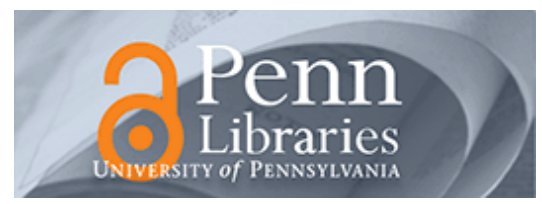

University of Pennsylvania ScholarlyCommons

3-15-2010

\title{
Double-diamond NaAl via Pressure: Understanding Structure Through Jones Zone Activation
}

Ji Feng

University of Pennsylvania, fengji@seas.upenn.edu

Roald Hoffmann

Cornell University

Neil Ashcroft

Cornell University

Follow this and additional works at: https://repository.upenn.edu/mse_papers

Part of the Materials Science and Engineering Commons

\section{Recommended Citation}

Feng, J., Hoffmann, R., \& Ashcroft, N. (2010). Double-diamond NaAl via Pressure: Understanding Structure Through Jones Zone Activation. Retrieved from https://repository.upenn.edu/mse_papers/178

Suggested Citation:

Feng, J., R. Hoffmann and N.W. Ashcroft. (2010). "Double-diamond NaAl via Pressure: Understanding Structure Through Jones Zone Activation." The Journal of Chemical Physics. 132, 114106.

(C) 2010 American Institute of Physics. This article may be downloaded for personal use only. Any other use requires prior permission of the author and the American Institute of Physics.

The following article appeared in The Journal of Chemical Physics and may be found at http://dx.doi.org/10.1063/ 1.3328198

This paper is posted at ScholarlyCommons. https://repository.upenn.edu/mse_papers/178

For more information, please contact repository@pobox.upenn.edu. 


\title{
Double-diamond NaAl via Pressure: Understanding Structure Through Jones Zone Activation
}

\author{
Abstract \\ Under normal conditions, sodium forms a 1:1 stoichiometric compound with indium, and also with \\ thallium, both in the double-diamond structure. But sodium does not combine with aluminum at all. Could \\ $\mathrm{NaAl}$ exist? If so, under what conditions and in which structural types? Instead of beginning with a purely \\ computational and first-principles structure search, we are led to apply the early Brillouin and higher \\ (Jones) zone ideas of the physics determining structural selection. We begin with a brief recapitulation of \\ the higher zone concept as applied to the stability of metals and intermetallic compounds. We then \\ discuss the extension of this concept to problems where density becomes a primary variable, within the \\ second-order band structure approximation. An analysis of the range of applicability of pressure-induced \\ Jones zone activation is presented. The simple NaAl compound serves us as a numerical laboratory for \\ the application of this concept. Higher zone arguments and chemical intuition lead quite naturally to the \\ suggestion that 1:1 compound formation between sodium and aluminum should be favored under \\ pressure and specifically in the double-diamond structure. This is confirmed computationally by density \\ functional theoretic methods within the generalized gradient approximation.

\section{Disciplines \\ Engineering | Materials Science and Engineering}

\section{Comments} \\ Suggested Citation: \\ Feng, J., R. Hoffmann and N.W. Ashcroft. (2010). "Double-diamond NaAl via Pressure: Understanding \\ Structure Through Jones Zone Activation." The Journal of Chemical Physics. 132, 114106. \\ (C) 2010 American Institute of Physics. This article may be downloaded for personal use only. Any other \\ use requires prior permission of the author and the American Institute of Physics. \\ The following article appeared in The Journal of Chemical Physics and may be found at http://dx.doi.org/ \\ $10.1063 / 1.3328198$
}




\title{
Double-diamond NaAl via pressure: Understanding structure through Jones zone activation
}

\author{
Ji Feng, ${ }^{1}$ Roald Hoffmann, ${ }^{2, a)}$ and N. W. Ashcroft ${ }^{3}$ \\ ${ }^{1}$ Department of Materials Science and Engineering, School of Engineering and Applied Science, \\ University of Pennsylvania, Philadelphia, Pennsylvania 19104, USA \\ ${ }^{2}$ Department of Chemistry and Chemical Biology, Baker Laboratory, Cornell University, Ithaca, \\ New York 14853-1301, USA \\ ${ }^{3}$ Laboratory of Atomic and Solid State Physics and Cornell Center for Materials Research, Clark Hall, \\ Cornell University, Ithaca, New York 14853-2501, USA
}

(Received 8 September 2009; accepted 2 February 2010; published online 15 March 2010)

\begin{abstract}
Under normal conditions, sodium forms a 1:1 stoichiometric compound with indium, and also with thallium, both in the double-diamond structure. But sodium does not combine with aluminum at all. Could $\mathrm{NaAl}$ exist? If so, under what conditions and in which structural types? Instead of beginning with a purely computational and first-principles structure search, we are led to apply the early Brillouin and higher (Jones) zone ideas of the physics determining structural selection. We begin with a brief recapitulation of the higher zone concept as applied to the stability of metals and intermetallic compounds. We then discuss the extension of this concept to problems where density becomes a primary variable, within the second-order band structure approximation. An analysis of the range of applicability of pressure-induced Jones zone activation is presented. The simple $\mathrm{NaAl}$ compound serves us as a numerical laboratory for the application of this concept. Higher zone arguments and chemical intuition lead quite naturally to the suggestion that 1:1 compound formation between sodium and aluminum should be favored under pressure and specifically in the double-diamond structure. This is confirmed computationally by density functional theoretic methods within the generalized gradient approximation. () 2010 American Institute of Physics.

[doi:10.1063/1.3328198]
\end{abstract}

Increase of density can cause pronounced changes in geometric arrangements and electronic structures in the condensed states of metals. In particular, metals traditionally considered "simple" have recently been shown to exhibit extraordinary structural variety and properties under high pressure, patently belying this characterization. For instance, lithium and sodium, or their combination with a second element, have been shown to undergo intricate structural transformations under pressure, in both liquid and solid phases, departing in fascinating ways from close packing. ${ }^{1-8}$ The dramatic change in reactivity and emergence of new compounds under pressure provide a productive arena for reexamining and deepening our understanding of the underlying structural principles. ${ }^{7-10}$ Obvious questions arise in this context: what new crystalline compounds might form under pressure and in what structures? And how might we act on clues before we actually begin any experimental synthesis or even before embarking on more extended first-principles calculations? And further, are structural principles developed for compounds at ordinary pressures still applicable when high pressure becomes the thermodynamic variable of choice? If so, then why?

A number of algorithms have been applied, with some success, to predict structures under high pressure or to complement experimental structure determination., ${ }^{7,8,11-13}$ However, these methods generally offer little physical insight

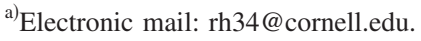

on why a particular configuration is favored or what reduced parameter space is pertinent to the structure of a class of materials under pressure. To set the problem in context, we note that a synthetic inorganic chemist, well versed in valence rules, is much less frequently bothered by the question "what can be made?," than by "how to make it?" And the chemist usually does make it, without recourse to a sophisticated quantum mechanical computational package. In stark contrast, however, it is a potent challenge to predict what is going to happen when we put the squeeze on a metal, or two of them, in a diamond anvil cell. The key issue here is the lack of qualitative understanding, call it insight or theory, relating the stability of metals and intermetallic compounds to a simple set of physical variables, especially when pressure is in the multigigapascal regime.

Some general trends concerning the crystalline metallic state of simple metals under pressure have become clear, and these may offer some guideposts for discovering new intermetallic phases. In particular, it has been pointed out, notably by Ackland and Macleod ${ }^{14}$ and by Degtyareva, ${ }^{15,16}$ that the Jones ${ }^{17}$ and later Mott and Jones ${ }^{18}$ stability arguments often play a critical role in determining structures in novel metallic phases under high pressure. ${ }^{7}$ The essence of this reasoning resides in an argument that the contact of bands with Brillouin zone (BZ) planes, associated with crystal (pseudo)potentials $V_{\mathbf{K}}$ (generally local), and states near the Fermi level can be a key stabilizing factor. The value of $V_{\mathbf{K}}$ varies in general in complex fashion with density, but straightforward 
computation of relevant $V_{\mathbf{K}}$ 's in simple forms may offer an efficient way of searching for stabilizing factors at high density. The argument for higher zones, including its implication for associated structural stability and instability, can be seen as an extension to the substantial and enriching complexities of three-dimensional (3D) structure of orbital overlap ${ }^{19}$ and also the Jahn-Teller effect, ${ }^{20}$ venerable molecular concepts of immense utility.

In this paper, we extend the higher zone concept to the context of intermetallic compounds under high pressure, following a brief retrospective of this concept for metals under ordinary conditions. Then we argue that elevation of average density can enhance the associated stability in metals and intermetallic compounds, through a mechanism that we call pressure-induced zone plane activation. Subsequently, we exemplify the utility of this concept by a computational investigation, guided by the higher zone reasoning (and supplemented with structural maps and a moderately automated structural search method) on the existence of binary intermetallic phases of sodium (Na) and aluminum (Al) under high pressure. Our results indicate that sodium and aluminum may be impelled to take up a 1:1 intermetallic compound by quite moderate pressure (just $12 \mathrm{GPa}$ ). The ensuing compound $\mathrm{NaAl}$ also adopts the sodium thallide (NaTl) structure, epitomized by the elegant double-diamond arrangement. The computational methodologies we use are described in detail in the Appendix.

\section{THE CONCEPT OF STABILITY WITHIN THE HIGHER (JONES) ZONE FRAMEWORK}

We begin our discussions of the Jones (and later Mott and Jones) arguments on the stability of certain intermetallic phases with a short review which is not meant to be exhaustive. Its purpose is to recapitulate the essence of this important formalism that we shall apply to structural problems at high pressure, specifically, in the $\mathrm{Na}-\mathrm{Al}$ example to follow.

From late 1920 s through the 1930 s, Hume-Rothery ${ }^{21}$ proposed empirical rules that for normal conditions of one atmosphere and moderate temperatures alloy solutions could undergo structural transitions at quite specific concentrations of their constituent metals. He further noted that disparities in ionic sizes should also be a contributing factor for overall stability. Viewing the special stability of specific concentration as a problem in which ions of suitably averaged valence continued to occupy the sites of one of the initiating lattices (with reciprocal lattice vectors $\{\mathbf{K}\}$ ), Jones ${ }^{17}$ and Mott and Jones $^{18}$ formulated a condition on the magnitude of the concentration-dependent free-electron Fermi wave vector, $k_{F}$, associated with such an instability, namely

$$
k_{F}=K / 2 \text {, }
$$

where the reciprocal lattice vector $\mathbf{K}$ involved was usually associated with the innermost plane of a particular zone. ${ }^{17,18}$ This is clearly a viewpoint rooted in the idea of a virtual crystal and also established within the independent-electron approximation.

The underlying notion was that alloys would change phase in such a way as to mainly accommodate the valence electrons within a certain zone, often the first but, important
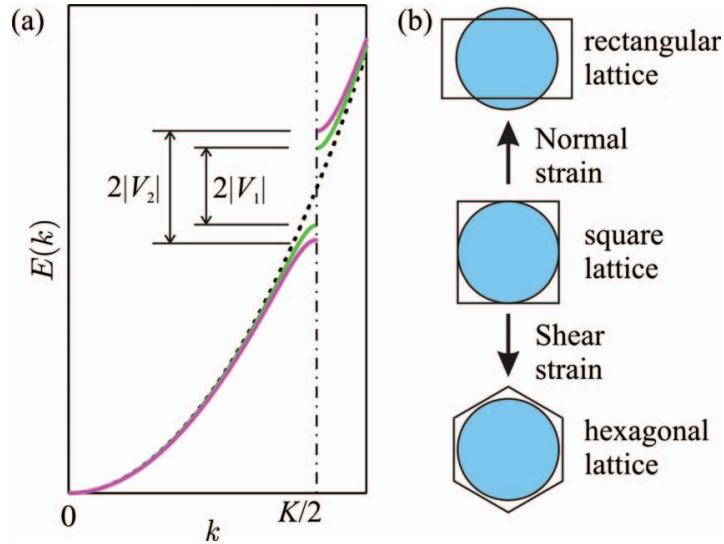

FIG. 1. The basic concept of the Jones and Mott and Jones' arguments for the Hume-Rothery systematization of stability. (a) Nearly free-electron bands in one dimension, with different lattice potential strengths at $\mathbf{K}\left(0, V_{1}\right.$, and $V_{2}$ ). (b) A 2D example of how the geometric structure affects the Mott and Jones type stability (or instability). We start with a 2D square lattice, whose first BZ is a square (shown as the square box), with electron count that the Fermi circle is just inscribed by the first BZ. Upon imposition of a normal or a predominantly shear strain, the first BZ becomes rectangular or hexagonal, shown as 2D boxes. The unit cell area (thus the area of the first $\mathrm{BZ}$ ) is kept constant. Note that the polygons are the first BZs, whose vertices are not necessarily reciprocal lattice points in general.

for what follows, also within higher zones (referred to often as the Jones zones ${ }^{22}$ ). Implicit in these ideas is a principle of involvement or contact of the Fermi surface of a hypothetical initial free-electron gas state with the planes of such a zone. The mechanism is clearly to be seen as part of a much wider approach to predicting the structures of metallic manyparticle systems, given the knowledge of the electron-ion interactions or pseudopotentials themselves. It should be noted that the pseudopotential $V(\mathbf{r})$ is itself related to Hume-Rothery's ${ }^{21}$ original conception of ion sizes.

An example of the stabilization being proposed is illustrated in Fig. 1(a), for a one-dimensional (1D) case. Starting with a free-electron band (which is gapless at the Bragg plane associated with $K / 2$ ), a band gap is opened as soon the lattice potential is turned on, providing the geometric structure factor associated with a possible basis is nonvanishing. Suppose that for this $1 \mathrm{D}$ system the electron count is such that free-electron levels are filled just up to $K / 2$. Then clearly a gap opening has a stabilizing effect on the electronic energy, and the overall stabilization will depend on the magnitude of $V(\mathbf{K})$ [see Fig. 1(a)].

It ought also to be noted that the stabilization energy (usually per atom) is defined as the energy of the final electronic system interacting with the ions, diminished by that of the starting uniform electronic system, at the same average electron density. It follows that it is intuitive and pragmatic to use the concept of emergent zone activation to compare the stability of different structures at the same electron count. But to determine the optimal electron count using Jones' condition [Eq. (1)] for a given structure will require more caution in establishing thermodynamic consistency.

The geometric implication of zone activation stability is actually apparent only when a problem in two or three dimensions is considered. As we show in Fig. 1(b), the geometric relation between BZ planes and the Fermi surface can 
be changed by structural rearrangement. In this hypothetical example, the Fermi circle is just inscribed in the square first BZ when the lattice itself is square [Fig. 1(b) middle]. Apparently, there is a topological interplay between the freeelectron Fermi surface and the shape of the BZ, where the latter becomes a variable as the lattice is subjected to a strain. For a general 3D crystal, the homogeneous strain can be characterized by a strain tensor, $\eta_{\alpha \beta}$, for which

$$
x_{\alpha, f}=\eta_{\alpha \beta} x_{\beta, i}
$$

where $\left\{x_{\alpha, i}\right\}$ and $\left\{x_{\alpha, f}\right\}$ are the initial and final atomic configurations, respectively, and $\alpha$ and $\beta$ represent $x, y$, and $z$ directions in Cartesian coordinates.

Keeping the electron count unchanged, we deform the structure by a normal strain and obtain a rectangular lattice. In this case, two of the first BZ planes intersect the freeelectron Fermi surface, while the other two separate from the Fermi surface [Fig. 1(b) top]. If we start with the square lattice again, but now apply a predominantly shear strain to form a hexagonal lattice (a small normal strain in addition to the shear is needed to form a hexagonal lattice), the Fermi surface will be entirely contained in the first BZ with a finite separation from the zone planes [Fig. 1(b) bottom]. Clearly, each of the structures in these elementary examples may in general be associated with quite different stabilizations because of the interaction between states near the zone planes. And those differences might in turn lead to a structural preference. In general, the polytope [a polygon in twodimensional (2D) and a polyhedron in 3D] formed by Bragg planes bounding the Fermi surface need not be the first BZ, but can be any polytope formed by Bragg planes that have the strongest contact with the Fermi sphere. In this context, the zone is called a Jones zone as introduced above.

There will surely be correspondingly induced electronic charge densities. Blandin ${ }^{23}$ and Heine ${ }^{24}$ both remarked upon the possibility that in reciprocal space rapid changes in electronic response densities near $2 k_{F}$ (in reciprocal space) might well be part of the impending physics of structural instability. This was confirmed in detail by Stroud and Ashcroft ${ }^{25,26}$ by direct evaluation of response or band structure energies, but they also noted that to account for the overall requirements of thermodynamic stability, it would be necessary to evaluate the response beyond linear order. The eventual structure will be one that minimizes the Gibbs energy, an important contributor to which is the electronic component determined in the field of the ions. In the mean field, independent-electron approximation, the Gibbs energy per electron of this component is the corresponding electronic chemical potential which, in the $T \rightarrow 0$ limit, becomes the Fermi energy for a metallic state. Though other terms are also of considerable structural importance (for example, electrostatic or Madelung contributions), this argues for ionic arrangements which, relative to band minima, actually lead to the lowest Fermi energies in static ground states. For the case where the initial unperturbed Fermi surface is within a zone plane (associated with $\mathbf{K})$, it has been shown ${ }^{27}$ that if $V(\mathbf{K})$ is the Fourier component of the associated pseudopotential, then from direct evaluation of the Fermi surface distortions caused by the zone plane, and the obvious constraint on the total $\mathbf{k}$-space volume enclosed by the Fermi surface) there is a reduction in Fermi energy which is proportional to

$$
\sum_{\mathbf{K}} V^{2}(\mathbf{y}) y \ln \left|\frac{1-y}{1+y}\right|,
$$

with $y=K / 2 k_{F}$. (This is relative to the free-electron Fermi level, $E_{F 0}$.) It is clear that the $y$-dependent term in Eq. (3) has a divergence, that is, $\ln |(1-y) /(1+y)| \rightarrow-\infty$ at $y=1$. Therefore, providing there is not too much reduction in $V(\mathbf{K})$ resulting from geometric structure factors, Eq. (3) manifestly suggests that a tendency toward intersection with the Jones zone, as well as potentially increasing values of $V(\mathbf{K})$, could then be favored in eventual structural selection. As a consequence, a zone plane therefore attains an effective width in $\mathbf{k}$ space related to the issue of energy lowering. It is defined through the observation that when the unperturbed Fermi energy comes within a range defined approximately by a typical lower zone plane energy splitting of $E_{0}(\mathbf{K} / 2)$ $-|V(\mathbf{K})|$, where as noted $V(\mathbf{K})$ may include a geometric structure factor when the lattice is decorated by a basis. ${ }^{28}$

In the elementary 1D example shown in Fig. 1(a), it is energetically beneficial to for the free-electron states near the Fermi level to scatter around $K / 2$ with $k$ systematically increasing toward $K / 2$, or at energies lower than those corresponding to the Bragg plane energy $E_{0}(\mathbf{K} / 2)$, as we see also from the form of the lower nearly free-electron band, $E_{0}(\mathbf{K} / 2)-|V(\mathbf{K})|$. But in principle, a structure could be chosen (with a smaller $K$ ) such that what is important is the upper band, starting at $E_{0}(\mathbf{K} / 2)+|V(\mathbf{K})|$, i.e., there is zone activation with $\mathrm{k}$ systematically decreasing toward $K / 2$. In 2D and 3D problems, when the free-electron Fermi circle or sphere intersects with a zone plane, zone contact can occur in principle from both below and above. Since the density of states (DOS) peaks at $E_{0}(\mathbf{K} / 2)-|V(\mathbf{K})|$ and then drops almost linearly from then on to $E_{0}(\mathbf{K} / 2)+|V(\mathbf{K})|$, the advantage of placing more electrons at lower energies will not necessarily be sustained, so that structural choices leading to contact of Bragg planes from below will remain the more favorable initial option. ${ }^{29}$

Although the chain of arguments starts with the HumeRothery's cogent observations on the systematics of alloying, at least in relatively simple systems, the links between the Fermi wavevector and the emerging structures are now considerably more intertwined. ${ }^{30}$ It is important to restate that these links involve electronic response to all orders and that the reference state is the hypothetical, initially free, electron gas prior to the introduction of an array of ions. However, it is also apparent that the zone plane set (and hence the realspace structure) chosen to lead to a significant (or "effective" in the sense described above) stabilization might well also lead to a preliminary, yet still useful, selection criterion for eventual structural choice.

A useful distinction can also be drawn between structural transitions arising from the Mott-Jones arguments as outlined above and those associated with the familiar Peierls mechanism. The underlying physics of both originates with manifestation of the periodic potential in terms of its effects on states near the highest occupied energy (the Fermi en- 
ergy). In the former case (zone activation), the initiating structure is potentially susceptible toward a deformation involving the entire unit cell. This can be seen as an example of a translationengleiche transformation, ${ }^{31}$ where a basic translational symmetry is preserved (and the reciprocal lattice vectors are also preserved), but a point group symmetry is at least partially lost. But in the latter, referred to often as the Peierls instability (and analyzed frequently through Fermi surface nesting arguments), the associated structural rearrangement usually leads to a supercell of the initiating structure. This can be seen as an example of a klassengeleiche transformation, where there is emergence of new reciprocal lattice vectors whose magnitudes are well suited to induce energy lowering (and stabilizing) distortions via states near the Fermi level.

\section{A HIGH-PRESSURE EXTENSION OF ZONE ACTIVATION INDUCED STABILITY}

Since $V(\mathbf{K})$ depends on the overall density of the system, the same question can be raised again, first for an element (or one component system) but under conditions where pressure $p$, and to a much lesser degree, temperature $T$, become the essential thermodynamic variables. In what follows, the reference system will be the uniform interacting electron gas and we introduce the standard linear measure, $r_{s}$, defined by $\Omega / N=4 \pi\left(r_{s} a_{o}\right)^{3} / 3$, where $\Omega$ is the volume of a unit cell, $N$ is the number of valence electrons in a unit cell, and $a_{o}$ the Bohr radius. Going a step further, the arguments below can also be extended to apply to ordered stoichiometric alloys, with their own possibly more complex zones, leading to the question: might such ordered alloys acquire their structures through the action of an "effectiveness" principle for zone planes that once again approach within a certain range of the free-electron Fermi surface, this range being highly dependent on the pseudopotentials? The number of such planes will be of critical importance and is obviously related to the issue of emergent complexity. Since the $V(\mathbf{K})$ 's can be quite dependent on $r_{s}$, the involvement of pressure through the Gibbs energy in structural choice and unit cell content is then immediate. It should be noted that the discussion next presented is limited to systems with reasonably simple electronic structures and not subject to, for example, pressure impelled $s-d$ or similar transfers (a point we return to below).

With pressure induced density dependence arising both via the energetics of the underlying nearly free-electron system but also through $V(\mathbf{K})$, structural changes can easily be envisaged in the alloying process these leading to the satisfaction of the zone-activation condition for arrangements other than the initiating structure. Evidently, the size of the zone (and whether what is now relevant is a Jones zone) is of considerable importance in the sum appearing in Eq. (3). And again, it is otherwise tied to a notion of zone planes becoming effective. Historically, it has also been seen via its manifestation in pair potentials, mainly through their major Fourier components when viewed in reciprocal space. ${ }^{32}$

In order to examine the issue of the density dependence of zone activation in the static lattice context, we reformulate the problem a little as follows. The system at hand is a neu- tral macroscopic assembly of interacting ions and, as stated above, interacting valence electrons, both systems obviously being coupled. To the valence electron system, we therefore add a uniform positive neutralizing background and to the ions a background of identical density but opposite in charge. Clearly the two charged continua thus introduced can be seen to cancel and thus do not affect the initiating problem. However, by formally preserving them, this construction sets up the familiar neutral interacting electron gas problem, with well defined thermodynamic functions. And it also sets up the problem of interacting but finite sized ions in a compensating background, also thermodynamically well defined, and a problem which is dominated by the Madelung energy. Finally the construction also sets up an interaction between electron and ionic charge distributions each, importantly, defined relative to their respective average densities.

Accordingly, denote the ground state energy per electron of the neutral interacting electron gas, as defined above, by $E_{\text {eg }}\left(r_{s}\right)$; we write this per electron in the standard way as

$$
E_{\mathrm{eg}}\left(r_{s}\right)=E_{\mathrm{ke}}\left(r_{s}\right)+E_{\mathrm{ex}}\left(r_{s}\right)+E_{\mathrm{corr}}\left(r_{s}\right),
$$

where the kinetic, exchange contributions electron (given in Rydbergs) are

$$
\frac{3}{5} \frac{(9 \pi / 4)^{2 / 3}}{r_{s}^{2}}
$$

and

$$
-\frac{3}{2 \pi} \frac{(9 \pi / 4)^{2 / 3}}{r_{s}},
$$

respectively. The correlation energy, $E_{\text {corr }}$, is small for the relatively free-electron-like systems we shall be considering and will be omitted in what follows.

For the ion-plus-background ground state equivalent, the static lattice assumption leads to the Madelung energy as a dominant term but necessarily corrected by the finite ion-size terms. The latter also enters into the electron-ion contributions in the long wavelength limit (see below). The Madelung energy per electron has the usual form

$$
E_{m}=-\frac{M}{r_{s}},
$$

where $M$ clearly depends on structure but weakly so. To be specific, if $9 / 5(1.8)$ is the ion-sphere value, ${ }^{33}$ then $M=9 / 5$ $-\delta M$, where $\delta M=0.00814$ for bcc, 0.00828 for fcc, 0.040 for sc, and 0.00832 for hcp (and in fact 0.021 for hexagonal, but with $c / a=2.00)$. This reflects the physical fact that, in a charged system, most of the energy resides at long range, where structural issues then recede. From the discussion above, the energy resulting from the interaction of the itinerant electrons with the ions (in the presence of the mutually neutralizing backgrounds) is just the quantity we refer to, per electron, as $E_{\mathrm{bs}}\left(r_{s}\right)$. Viewed as a perturbation, it corrects the uniform interacting electron gas energy formally to all orders.

As a consequence, the total energy, per electron, of the metallic system is 


$$
E\left(r_{s}\right)=E_{\mathrm{eg}}\left(r_{s}\right)+E_{m}\left(r_{s}\right)+E_{K \rightarrow 0}\left(r_{s}\right)+E_{\mathrm{bs}}\left(r_{s}\right) .
$$

Here $E_{K \rightarrow 0}$ is the long wavelength limit of electron-ion and ion-ion interactions, and it is dependent on the character of the pseudopotential. By way of example, for the empty-core pseudopotential, characterized by a core dimension $r_{c}$, it takes the simple form $3 r_{c}^{2} / r_{s}^{3}$, per electron and it is a significant energy. From Eq. (4), the issue of structural preference now focuses immediately on its last two terms but in fact, mainly on the last in view of the above. For high pressure experiments, what is quite essential is the Gibbs energy, per electron, and under the stated conditions of low temperature this is

$$
\begin{aligned}
\frac{G}{N}=E+\frac{p V}{N}= & E-\frac{1}{3 r_{s}} \frac{d E}{d r_{s}} \\
= & E_{F 0}\left(r_{s}\right)+\frac{4}{3} E_{\mathrm{ex}}\left(r_{s}\right)+\frac{4}{3} E_{m}\left(r_{s}\right)+E_{K \rightarrow 0}\left(r_{s}\right) \\
& \times\left[1+\frac{d\left|E_{K \rightarrow 0}\left(r_{s}\right)\right|}{d \ln n}\right] \\
& +E_{\mathrm{bs}}\left(r_{s}\right)\left[1+\frac{d E_{\mathrm{bs}}\left(r_{s}\right)}{d \ln n}\right]
\end{aligned}
$$

where, and as is to be expected, the first term, $E_{F 0}\left(r_{s}\right)$, in $G / N$ is the zero temperature limit of the chemical potential of a noninteracting Fermion system, the Fermi energy. For the empty-core pseudopotential, again as an example, the second line in Eq. (5) is just $12 r_{c}^{2} / r_{s}^{3}$ and is structure independent. ${ }^{34}$ It is clear that the last line is then dominated by $E_{\mathrm{bs}}$ itself. Note that the pressure is

$$
p=-\left(4 \pi r_{s}^{2}\right) \frac{d E}{d r_{s}},
$$

from which $r_{s}$ will follow straightforwardly for a given $p$ (the atomic unit of $e^{2} / 2 a_{o}^{4}$, or equivalently, $14720 \mathrm{GPa}$ ).

The possible preference for higher zone introduction can now be illustrated for the specific case where band structure energies are reasonably given within linear response. There are surely systems where band structure energies may be dominated by induced electron densities originating with quadratic and higher response in the electron gas (leading to three-body and higher effective ion-ion interactions). As cautioned above, the zone-activation arguments will not necessarily hold for these cases. But to illustrate the role of emergent zone plane complexity further, let us first define wavevectors using density independent quantities; thus let $\mathbf{y}$ $=\mathbf{k} / 2 k_{F}$ and $\mathbf{Y}=\mathbf{K} / 2 k_{F}$, where $k_{F}$ is magnitude of the Fermi wave vector and $\mathbf{K}$ is a reciprocal lattice vector appropriate to a chosen structure. To second order in pseudopotentials, the band structure energy is then given by the well known linear response result which (in Rydbergs per electron) is ${ }^{28,35}$

$$
E_{\mathrm{bs}}=\sum_{\mathbf{Y} \neq 0} E_{\mathrm{bs}}\left(\mathbf{Y}, r_{s}\right)=c r_{s} \sum_{\mathbf{Y} \neq 0} Y^{2} V\left(\mathbf{Y}, r_{s}\right)^{2}\left[\varepsilon\left(\mathbf{Y}, r_{s}\right)^{-1}-1\right],
$$

where

$$
E_{\mathrm{bs}}\left(\mathbf{Y}, r_{s}\right)=c r_{s} Y^{2} V\left(\mathbf{Y}, r_{s}\right)^{2}\left[\varepsilon\left(\mathbf{Y}, r_{s}\right)^{-1}-1\right],
$$

Here $c \equiv 12^{1 / 3} \pi^{2 / 3}$ when atomic units are used, and $\varepsilon\left(\mathbf{Y}, r_{s}\right)$ is the electronic dielectric function. In this approximation, $E_{\mathrm{bs}}\left(r_{s}\right)$ is negative definite, which is of interest to the issue of its subsequent changes. Neglecting, for the moment, further corrections for exchange and correlation, epsilon is taken to have the familiar Lindhard form for the homogeneous electron gas, namely

$$
\varepsilon(Y)=1+\frac{r_{s}}{6.0292 Y^{2}}\left[\frac{1}{2}+\frac{1-Y^{2}}{4 Y} \ln \left|\frac{1-Y}{1+Y}\right|\right],
$$

In chemical terms, this band structure energy [Eq. (4)] can generally be thought of as a sum of bonding energies these originating with "unbound orbitals," which are taken as extended free-electron states, which also subsequently "interact" in an effective way through matrix elements of the form $\langle\mathbf{k}|V(\mathbf{r})| \mathbf{k}+\mathbf{K}\rangle$. To leading order the electron-electron interactions are being accounted for through the random phase approximation (RPA), and as stated $\varepsilon\left(\mathbf{Y}, r_{s}\right)$ is the electronic dielectric function.

From inspection of Eqs. (7a) and (8), it is immediately evident that for a given static ionic arrangement, the band structure energy clearly depends of course on $r_{s}$ (and through it, $k_{F}$ ), but just as clearly on the set of all reciprocal lattice vectors $\{\mathbf{K}\}$ appropriate to the structure. It will also depend on any possible basis vectors for non-Bravais lattices. To elucidate the dependence of $E_{\mathrm{bs}}$ on a change in $r_{s}$ impelled by pressure, we observe that $d Y / d r_{s}=0$, the rate of change of each $Y$ component of the band structure energy with $r_{s}$ is then

$\left[\frac{\partial E_{\mathrm{bs}}(\mathbf{Y})}{\partial r_{s}}\right]_{T}=\frac{E_{\mathrm{bs}}(\mathbf{Y})}{r_{s}}\left[1+\varepsilon(Y)^{-1}+\frac{2}{|V(\mathbf{K})|} \frac{d\left|V\left(\mathbf{Y}, r_{s}\right)\right|}{d r_{s}}\right]$.

Since, as noted, $E_{\mathrm{bs}}$ is negative, it follows that at the level of linear response, the sign of this result hinges on the rate of change of the $\left|V\left(\mathbf{Y}, r_{s}\right)\right|$, and the issue of stability then very much devolves on the chemical character of the constituent atoms. But if $d E_{\mathrm{bs}} / d r_{s}>0$, a structure may ensue which can be stabilized under progressive compression simply through the appearance of further zone planes and their associated $V(\mathbf{K})$ 's. Put another way, with $\mathbf{K}$ in the vicinity of $2 k_{F}$, significant values of $V(\mathbf{K})$ may appear (the associated Bragg planes forming a Jones zones enveloping the free-electron sphere) even though at lower pressure such structures may not be stable. This is what we may refer to as pressureinduced Jones zone activation. Note once again that if the physical system and its chemical characterization are such that linear response is insufficient, the activation of zone planes is by no means guaranteed.

Finally, it is worth re-emphasizing that rapid variation of the dielectric function in the vicinity of $2 k_{F}$ plays a crucial role $^{25}$ in the concentration rule for alloys (Hume-Rothery's original systematization) when overall density is not significantly altered. Through continuous alloying it is possible to vary $2 k_{F}$ to match optimally with $\mathrm{K}$. Here, and in contrast to this, the density variations are such that $\mathbf{K} / 2 k_{F}$ remains 


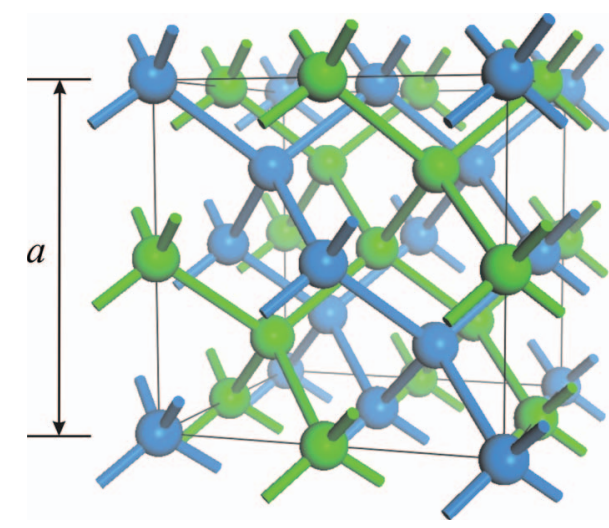

FIG. 2. A cubic unit cell rendition of the double-diamond structure. This structure consists of two sublattices, and each forms a diamond network (blue and green). In this structure, each atom sits in the center of a cubic cage, with four like atoms on one set of the tetrahedral vertices, and four atoms of the other type on the remaining set of tetrahedral vertices of the cubic cage. A full unit cells consists of eight of such cubic cages, with a total of eight of atoms of each type. It can also be viewed as a face centered cubic with a four-point basis.

largely constant for hydrostatic strains. The rapid changes in the dielectric function remain of key importance.

\section{Na-AI UNDER PRESSURE: A CASE STUDY}

We now demonstrate the utility of the zone plane activation argument in finding novel potentially ordered alloy systems under pressure. By itself, the argument cannot point directly to which new compounds might form under pressure. However, what led us first to NaAl was simply chemical reasoning through analogy. Subsequently, zone plane activation thinking comes into play and markedly helps us to see the possibility of forming a NaAl alloy, of a certain very interesting structure, under pressure.

\section{A. Chemical analogy}

Under standard conditions, sodium forms a 1:1 stoichiometric compound with indium and also with thallium, both of these in the double-diamond structure, as does the compound of lithium with aluminum. This elegant structure is composed of two interpenetrating diamond networks (see Fig. 2), each accommodating a single element. NaTl is the original Zintl phase, the progenitor of a tremendously useful heuristic for looking at the structures of binary and ternary compounds of electropositive atoms and main group elements. ${ }^{36,37}$ The structure of $\mathrm{KTl}$ at one atmosphere is another type of Zintl compound, containing octahedral $\mathrm{Tl}_{6}^{6-}$ clusters. $^{38}$ At elevated pressures, KTl goes into the $\mathrm{NaTl}$ structure too. ${ }^{39}$

On the other hand, sodium and aluminum are not known to combine at ordinary pressures and temperatures. ${ }^{40}$ But the possibility that these two elements may form compound(s) under elevated pressure is as yet unexplored. ${ }^{41}$ The existence of other compounds between groups 1 and 13 in the doublediamond structure is an indication that zone plane activation and stabilization may also be at work in these compounds, with the given electron count and structure. It follows then that for the $1: 1$ combination of $\mathrm{Na}$ and $\mathrm{Al}$, the double- diamond structure may well be susceptible to such stabilization, only awaiting activation by external pressure.

The existence of such a compound is potentially of considerable interest from another perspective. The known ternary systems of $\mathrm{Na}-\mathrm{Al}-\mathrm{H}$ are important candidates for hydrogen storage. The adsorption and desorption of hydrogen follow a rather complex pathway involving $\mathrm{NaAlH}_{4}$, $\mathrm{Na}_{3} \mathrm{AlH}_{6}, \mathrm{NaH}, \mathrm{H}_{2}$, and Al. In a recent calculation, ${ }^{42}$ it is found in the chemically similar $\mathrm{Li}-\mathrm{Al}-\mathrm{H}$ system, that the enthalpy of the reaction $\mathrm{LiH}+\mathrm{Al}=\mathrm{LiAl}+\frac{1}{2} \mathrm{H}_{2}$ is in fact negative under standard conditions. One expects that a similar reaction would be favored if the enthalpy of formation for $\mathrm{NaAl}$ itself could be substantially reduced under compression. The existence of $\mathrm{Na}-\mathrm{Al}$ intermetallic compounds, as additional intermediates in these cycles, may potentially provide alternative pathways that can influence hydrogen release and the reversibility of the chemical cycle.

We are then naturally intrigued by a very pertinent question, namely, whether the double-diamond structure is also a possibility for $\mathrm{Na}$ and $\mathrm{Al}$ compound formation, but now under pressure. In the present work, we have limited ourselves to $1: 1$ stoichiometry. There may well be other stoichiometries that become stable at high pressure, but (1) the 1:1 stoichiometry is the simplest, yet is very common among intermetallic compounds; (2) the focal point of our paper is actually to provide a proof of principle for the zone plane activation arguments (and chemical intuition) in guiding the search for possible new intermetallic phases under high pressure; (3) and given the complexity of structural theory, there is as yet no practical method for surveying all stoichiometries and the associated configuration spaces exhaustively. Because of the chemical guidelines, the 1:1 NaAl stoichiometry emerges as a very compelling start.

\section{B. Jones and zone plane activation reasoning for $\mathrm{NaAl}$}

What immediately follows is a semiquantitative analysis of the stability of the possible $\mathrm{NaAl}$ phase in the doublediamond structure, based on the zone plane activation type of reasoning. The space group and associated Laue symmetry of the double-diamond structure type suggest that the first nonvanishing components of the lattice potential are for reciprocal lattice vectors $\mathbf{K}=(111)$, (220), and (311). A Bragg plane associated with $\mathbf{K}$ is the plane in reciprocal space perpendicularly bisecting the vector $\mathbf{K}$, at a distance $K / 2=\left(h^{2}\right.$ $\left.+k^{2}+l^{2}\right)^{1 / 2}(\pi / a)$ from the origin. For an electron count of four valence electrons per formula unit ( $\mathrm{LiAl}, \mathrm{NaTl}$, or $\mathrm{NaAl})$ the magnitude of the free-electron Fermi wavevector is $k_{F}=\left(3 \pi^{2} n\right)^{1 / 3}=1.563(2 \pi / a)$, where $a$ is the lattice parameter of the cubic unit cell (with the average electron density $n=32 / a^{3}$, see Fig. 2). Therefore, the first Jones zone is the polyhedron bounded by the (220) zone planes. ${ }^{18}$ The (220) zone is a rhombic dodecahedron with 14 vertices [Fig. 3(a)], which, as shown in Fig. 3(b), can be viewed as a stellation of a cube. The same picture of chemical bonding has been suggested for intermetallic phases in the double-diamond ( $\mathrm{NaTl}$ structure) (see, for example, Inglesfield ${ }^{43}$ and also McNeil et $\left.a l . .^{44}\right)$. 

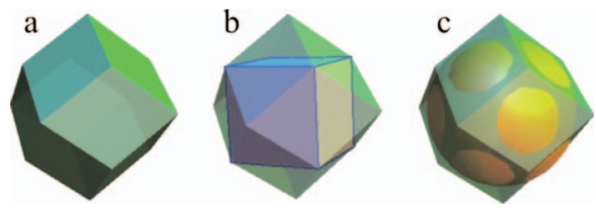

FIG. 3. (a) The (220) zone of the double-diamond structure type. (b) The (220) Jones zone is shown as a stellation of a cube. (c) The intersection of the (220) zone and the free-electron Fermi sphere (orange color), whose radius corresponds to the valence electron count of $\mathrm{LiAl}, \mathrm{NaTl}$, and the proposed $\mathrm{NaAl}$.

In Fig. 3(c), we show the (220) zone and the freeelectron Fermi sphere at the electron concentration of the double-diamond $\mathrm{NaAl}$ phase. Observe that the free-electron Fermi sphere intersects all faces of the (220) zone, as expected. Thus, the double-diamond phase fulfills the "geometric" requirement for maximizing the effectiveness of the higher zone activation mechanism. Its Fermi sphere is bounded by and even maximally fills ${ }^{45}$ the volume of a highly symmetric large zone and large numbers of states near the Fermi energy are then susceptible to zone plane perturbation.

The other condition required for stabilization within the zone plane activation framework is a strong lattice (pseudo)potential associated with the large set of zone planes. As noted in several recent articles, ${ }^{14,15} \mathrm{x}$-ray diffraction patterns are often useful in revealing the strengths of lattice potentials. Ackland and Macleod ${ }^{14}$ and Degtyareva, ${ }^{15,16}$ in particular, have productively explored this association. X-ray diffraction intensity is proportional to the square of the Fourier transform of the electron density, which for most elements resides largely within the cores. Simulated x-ray diffraction patterns indicate that for $\mathrm{LiAl}, \mathrm{NaTl}$, and $\mathrm{NaAl}$ in the double-diamond structure the (220) reflection has the strongest intensity (Fig. 4), (see comment above) lying slightly below twice the free-electron Fermi wavevector, $2 k_{F}$. This observation indicates that $V(220)$ is likely to contribute sub-

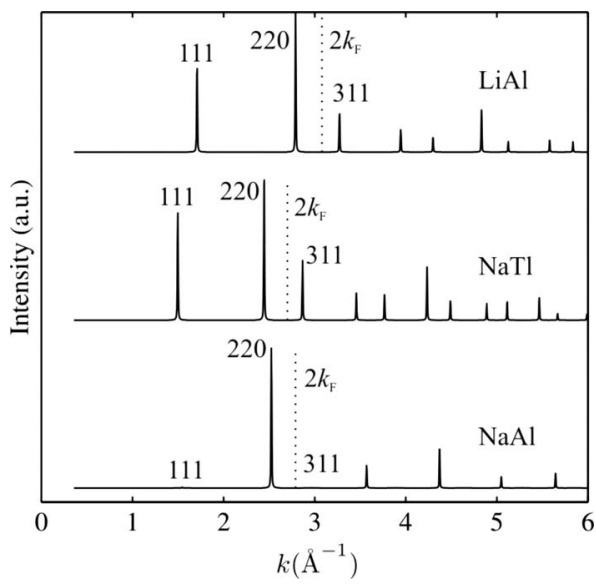

FIG. 4. Simulated diffraction patterns of LiAl, NaTl, and NaAl, all in the cF16 structure. The powder patterns are generated by PowderCell2.4 (a finite width is assigned to each peak to account for the finite sizes of crystallites in a diffraction experiment, and correspondingly, the peaks are not divergent Dirac-delta distributions) (Ref. 46). We use the DFT optimized equilibrium structure of $\mathrm{NaAl}(a=7.04 \AA$, see later). The experimental lattice parameters for $\mathrm{LiAl}(6.37 \AA)$ and $\mathrm{NaTl}(7.46 \AA)$ are used. The first three diffraction peaks are labeled with their indices.
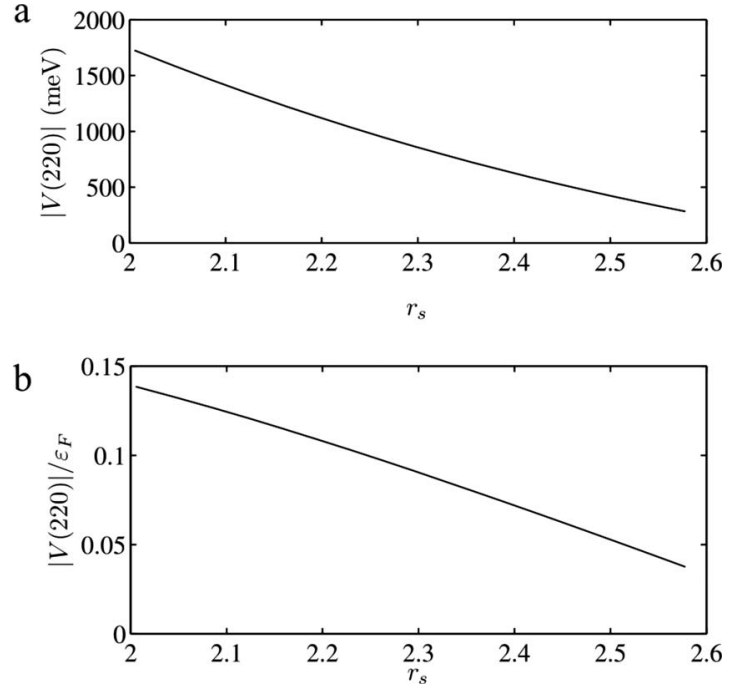

FIG. 5. How (a) $|V(220)|$ and (b) $|V(220)| / \varepsilon_{F}$ vary with $r_{s}$ (density increasing to the left). For this qualitative analysis, we use the empty core pseudopotential (Ref. 47), which has a form: $v_{\alpha}^{p s}(k)=-\left(4 \pi Z_{\alpha} / \Omega k^{2}\right) \cos \left(k R_{c, \alpha}\right)$, where $\alpha$ denotes the element, $Z_{\alpha}$ is the charge of the ionic core and $\Omega$ is the unit cell volume. The core radii, $R_{c}$, for $\mathrm{Na}$ and $\mathrm{Al}$ are 1.66 and $1.12 a_{o}$, respectively. In these plots, $r_{s}$ is given in units $a_{o} .|V(220)|$ is calculated for a 16-atom cubic unit cell. The lattice potential component is then $V_{\mathbf{K}}=\Omega^{-1} \Sigma_{\alpha} S_{\alpha}(\mathbf{K}) v_{\alpha}^{p s}(\mathbf{K})$, where $S_{\alpha}(\mathbf{K})$ is the geometric structure factor of ion $\alpha(\alpha=\mathrm{Na}$ and $\mathrm{Al})$.

stantially to the stability of the family of double-diamond compounds. The idea then suggests itself that one might gauge the existence of $\mathrm{NaAl}$ in the double-diamond structure by first assessing the magnitude of $|V(220)|$ and also how it varies with increasing density.

Accordingly, in Fig. 5(a), we plot the magnitude of $V(220)$ (Ref. 47) for $\mathrm{NaAl}$ in the double-diamond structure as a function of $r_{s}$, with $N=32$. With a moderate increase in density, i.e., a decrease in $r_{s}\left(\rho \propto r_{s}^{-3}\right)$, the magnitude of $V(220)$ increases almost fourfold. An even more revealing quantity is the ratio of $|V(220)|$ to the valence band width $\varepsilon_{F}$, which in the free-electron case is given by $E_{F}=50.1 \mathrm{eV} / r_{s}^{2}$. This quantity gives a measure of the importance of BZ plane perturbation as gauged by the overall band energy. As shown in Fig. 5(b), the value of $|V(220)| / E_{F}$ also increases dramatically with moderate increase in density.

In Fig. 6, we see that the overall second-order band structure energy from $\mathrm{NaAl}$ in the double-diamond structure becomes progressively more negative (hence more stabilizing) with increasing density. This suggests that $\mathrm{NaAl}$ may indeed be stabilized by pressure.

From the structure of Eq. (7b), it is readily seen that the band structure energy can be decomposed into selected contributions from $V(\mathbf{K})$ 's, as we also plot in Fig. 6. At one atmosphere $\left(r_{s} \sim 2.6\right)$, the band structure energy is dominated by the contribution from $V(111)$. Other $V$ 's for small $(h k l)$ have rather small contributions. As pressure and hence density rises, $E_{\mathrm{bs}}(111)$ is slightly increased (destabilizing), but concomitantly, $E_{\mathrm{bs}}(220)$ decreases rapidly by an amount roughly equal to the drop in overall $E_{\mathrm{bs}}$. The change in contributions from all other $\mathbf{K}$ 's is quite small compared to $E_{\mathrm{bs}}(220)$ and $E_{\mathrm{bs}}(111)$. Therefore, from the band structure energy analysis, pressure induced stabilization of $\mathrm{NaAl}$ in the 


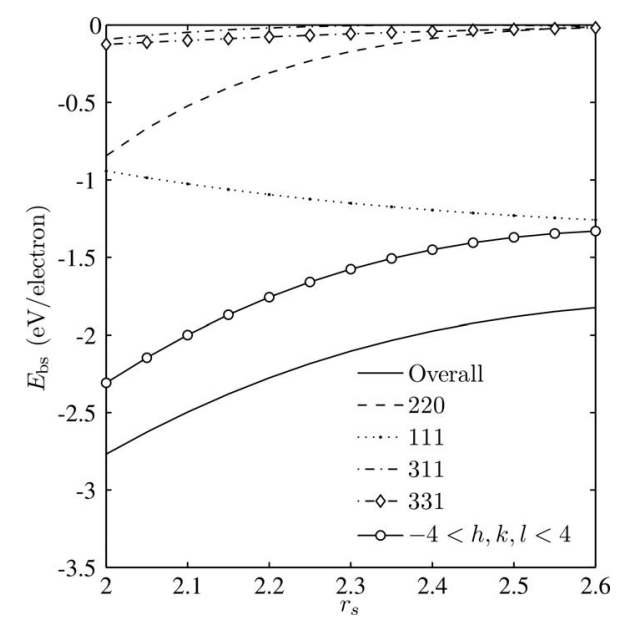

FIG. 6. Band structure energy, per electron, of $\mathrm{NaAl}$ (density increases to the left) in the double diamond structure, with the same pseudopotential used previously (Fig. 3) and with the Lindhard dielectric function. The overall band structure energy (solid line) and contributions from various BZ planes are plotted as function of $r_{s}\left(2.0<r_{s}<2.6\right)$. For a set of $h k l$ we sum over all distinct permutations $( \pm h \pm k \pm l)$, as they are equivalent because of the cubic symmetry. A partial sum for $-4<h, k, l<4$ (solid line with open circles) is also shown.

double-diamond structure should arise mainly from the contribution from $V(220)$, completely in accordance with zone plane activation reasoning above. The essential condition here is the closeness of the BZ planes to $2 k_{F}$. In the case of $\mathrm{NaAl}$ in the double-diamond structure, the (220) contribution indeed enjoys the greatest stabilization as density increases. The contributions from $-4<h, k, l<4$ differ from the overall $E_{\mathrm{bs}}$ in the $r_{s}$ range only by a roughly constant shift which indicates that the sum of contributions from zone planes associated with high $K$ 's, while appreciable, is fairly insensitive to density change.

A stability analysis of this sort is dependent upon the assumption that it is valid to use the simple pseudopotential from an independent electron approach for semiquantitative assessment. In fact, our calculation takes into account electron-electron interactions within the RPA (exchange and correlation contributions should not lead to significant corrections), and this matter will be addressed in the density functional approach to be described below. More importantly, we see from Eq. (6) that Brillouin and Jones zone activation by pressure should be present, arising largely from an increase in the density. In addition, we have demonstrated recently that the simple empty-core pseudopotential can be successfully used to explain some aspects of the structural complexity of $\mathrm{CaLi}_{2}$ under high pressure ${ }^{8,48}$ and can account for subtle features in the band structures derived from density functional theory (DFT) calculations. In spite of its simplicity, this analysis should therefore suffice for the initial argument in this context.

All of the observations above indicate that for $\mathrm{NaAl}$ in the double-diamond structure, the Jones zone planes become increasingly activated as the density increases, suggesting in turn an electronic stabilization of this phase by pressure. These arguments form the background of understanding of the structural problem which can then followed by accurate first principles calculations, to be described next.

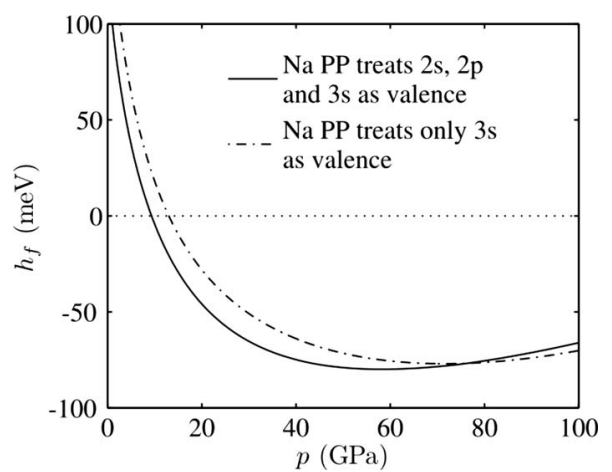

FIG. 7. Calculated enthalpy of formation per atom of NaAl from DFT-GGA, with and without the $2 s$ and $2 p$ electrons of $\mathrm{Na}$ in the self-consistent fields,, as different forms of pseudopotentials (PP) are employed (see Appendix). Pressure is obtained by numerically evaluating $p=-\partial E / \partial \Omega$, where $E$ is the energy per unit cell each with a volume $\Omega$. We expand the total energy with a polynomial of the form: $E=\Sigma_{m} \beta_{m} \Omega^{(m-2) / 3}$, where the sum is from $m=0$ to $m=6$.

\section{THE DOUBLE-DIAMOND NaAI PHASE AND DFT}

\section{A. DFT energetics}

Guided by the forgoing perturbation analysis, we performed DFT- generalized-gradient approximation (GGA) calculations to obtain an accurate $0 \mathrm{~K}$ enthalpy of formation of $\mathrm{NaAl}$ in the double-diamond structure. ${ }^{49}$ For an assumed static structure, we calculated the total energies with and without incorporation of the $2 s$ and $2 p$ electrons of sodium in the underlying pseudopotential. The calculated enthalpies are plotted as functions of pressure (Fig. 7). Both types of calculation yield completely similar trends. The enthalpy of formation $\left(h_{f}\right)$ of a potential $\mathrm{Na}-\mathrm{Al}$ alloy phase has the standard definition

$$
h_{f}\left[\mathrm{Na}_{x} \mathrm{Al}_{1-x}\right]=h\left[\mathrm{Na}_{x} \mathrm{Al}_{1-x}\right]-x h[\mathrm{Na}]-(1-x) h[\mathrm{Al}],
$$

where all enthalpies are given on a per-atom basis (and at the same pressure), and for $x=0.5$ in NaAl. The enthalpies of $\mathrm{Na}$ and $\mathrm{Al}$ are calculated for the more stable phases at a given pressure. It is known that $\mathrm{Na}$ undergoes a series of phase transitions in this pressure range, as bcc $\rightarrow \mathrm{fcc} \rightarrow \mathrm{cI} 16$ (and even more complex phases at higher pressure), while $\mathrm{Al}$ is quite stable in the fcc structure between 0 and $100 \mathrm{GPa}$.

We confirm that compound formation is not favored at one atmosphere and in the ground state, where the $h_{f}$ is well over $+100 \mathrm{meV} /$ atom, consistent with the fact that no compound has been observed between these two elements. But as pressure increases, the value of $h_{f}$ drops rapidly. However, we also find that at the relatively low pressure of $10 \mathrm{GPa}$ $\left(r_{s}=2.4\right)$, the reaction forming the compound turns neutral with respect to the separated elements, after which it becomes enthalpically preferred. At $55 \mathrm{GPa}, h_{f}$ passes through a minimum of $-80.0 \mathrm{meV} /$ atom. Subsequently, the value of $h_{f}$ increases slowly as the pressure rises toward $100 \mathrm{GPa}$, but still remains significantly negative.

The DFT calculations suggest that above $10 \mathrm{GPa} \mathrm{NaAl}$ ( $81 \%$ equilibrium volume) with the double-diamond structure is stable with respect to the elements. But is this the only structure likely? We have also performed an extensive struc- 

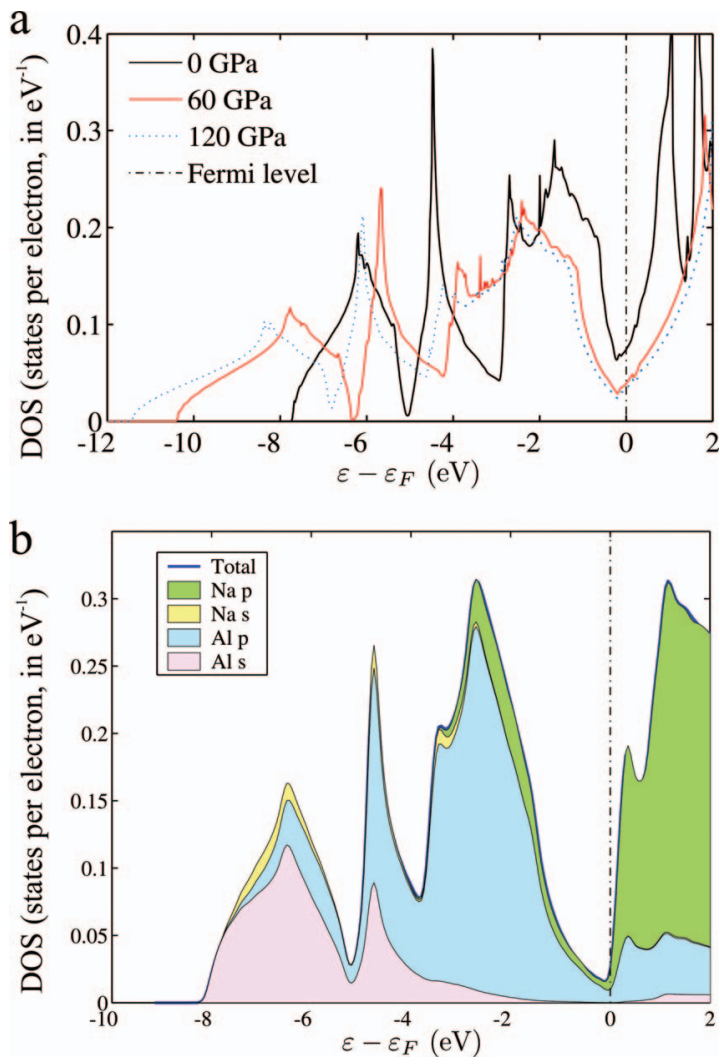

FIG. 8. (a) Valence Kohn-Sham electronic DOS per electron of the doublediamond phase at 0,60 , and $120 \mathrm{GPa}$. The energy scales have been shifted such that Fermi energies at all densities are at zero $\mathrm{eV}$ (the vertical dotdashed line). (b) DOS of NaAl (at one atmosphere) calculated with the extended Hückel method, with $s$ and $p$ projections for both $\mathrm{Na}$ and $\mathrm{Al}$.

tural search to identify other possibly competing structures for $\mathrm{NaAl}$ compounds. Our methodology includes both a random search method and the screening of structures suggested by Pettifor's binary structure map. ${ }^{12,50,51}$ We have also looked at the known KTl structure at $1 \mathrm{~atm}$. The structures explored are detailed in the auxiliary materials, ${ }^{50}$ but to date none have emerged as competitive when compared to the double-diamond structure. We therefore predict this ordered alloy can be fabricated under pressure. And the detailed calculations further support the general idea that the zone plane activation type of reasoning has significant value as a guide to proposing potential structures.

\section{B. Electronic structure of the double-diamond $\mathrm{NaAl}$ phase}

Since the proposed stabilization mechanism is electronic in origin, its presence should have a visible effect on the electronic structure of the $\mathrm{NaAl}$ compound as pressure increases, especially near the Fermi level. The matrix element, $\langle\mathbf{k}|V(\mathbf{r})| \mathbf{k}+\mathbf{K}\rangle$ should increase in magnitude for a favorable zone plane activation mechanism, leading to an increasingly large level splitting. Since this mechanism mainly influences the states near $E_{F}$, it is expected to result in a depletion of the electronic DOS around the Fermi level. In Fig. 8, we show the valence electronic DOS at 0,60 , and $120 \mathrm{GPa}$ for $\mathrm{NaAl}$ in the double-diamond structure.
TABLE I. Extended Hückel parameters for $\mathrm{Na}$ and $\mathrm{Al}$. The H's are the diagonal elements of the tight-binding Hamiltonian and $\xi$ 's are the exponents of the Slater-type orbitals. The off-diagonal elements are calculated according to $H_{i j}=\kappa S_{i j}\left(H_{i i}+H_{j j}\right) / 2$, where $S_{i j}$ is the overlap integral and $\kappa$ $=1.75$ is the Wolfsberg-Helmholtz mixing parameter.

\begin{tabular}{lcccc}
\hline \hline Element & $H(3 s) / \mathrm{eV}$ & $\xi(3 s) / \AA^{-1}$ & $H(2 p) / \mathrm{eV}$ & $\xi(2 p) / \AA^{-1}$ \\
\hline $\mathrm{Na}$ & -4.10 & 0.733 & -2.00 & 1.233 \\
$\mathrm{Al}$ & -7.30 & 1.167 & -4.50 & 0.967 \\
\hline \hline
\end{tabular}

Per electron, the DOS of the double-diamond phase has a prominent pseudogap near the Fermi level, indeed indicating a large pseudopotential at the large zone planes. This pseudogap is also evident in a number of calculations on NaTl. ${ }^{44,52-54}$ More significantly, with increasing pressure there is a manifestly progressive depletion of states around the Fermi level, as indicated by the increasingly wider and deeper pseudogap at $E_{F}$. From 0 to $60 \mathrm{GPa}$, the population of states at the Fermi level drops by nearly $50 \%$. The gap widening is much less visible as pressure increases from 60 to $120 \mathrm{GPa}$. There is also a persistent subvalence pseudogap (at $-5 \mathrm{eV}$ at $1 \mathrm{~atm},-6.5 \mathrm{eV}$ at $60 \mathrm{GPa}$ and $6.8 \mathrm{eV}$ at $120 \mathrm{GPa}$ ), which is mainly a manifestation of the strong level splitting, traceable to the presence of significant (111) component of the lattice potential (this can be readily deduced from the DFT-GGA band structure, see Ref. 50).

We notice the resemblance of the DOS of NaAl to those of $\mathrm{LiAl},{ }^{55} \mathrm{C}, \mathrm{Si}$, and $\mathrm{Ge}$, except that $\mathrm{NaAl}$ is metallic while $\mathrm{C}, \mathrm{Si}$, and $\mathrm{Ge}$ have band gaps under normal conditions. Given the relative electronegativities, it is likely that $\mathrm{Al}$ in $\mathrm{NaAl}$ is in an " $\mathrm{Al}^{-"}$ " configuration, with four valence electrons, in accord with a Zintl view of the double-diamond structure. ${ }^{36,37}$ This is the correct number to form a covalently bonded anionic diamond network that is isoelectronic to group 14 elements in the diamond structure. It is then useful to examine the character of bonding, and for this the simple extended Hückel method ${ }^{56}$ is ideal. We proceeded to parameterize the extended Hückel model to yield a DOS for NaAl in the double-diamond structure, and this qualitatively resembles the Kohn-Sham DOS [Fig. 8(b)]. The extended Hückel parameters are listed in Table I.

From these extended Hückel calculations, we see that the gap at $-5.2 \mathrm{eV}$ separates $s$ and $s p$ manifolds. The lowest manifold (from -8 to $-5.2 \mathrm{eV}$ ) is dominated by $s$ states of $\mathrm{Al}$, with small contributions from $\mathrm{Al} p$ and to a much lesser extent, $s$ of Na. The calculated maximum at $-6.5 \mathrm{eV}$ coincides with the transition of the character these orbitals from being bonding to antibonding, judging from the overlap population analysis ${ }^{57}$ (not shown). The $s p$ manifold (from -5.2 to $-3.8 \mathrm{eV}$ ) again has a maximum that coincides with the transition from antibonding to bonding characters. Above $-3.8 \mathrm{eV}$ and up to the Fermi level, the orbitals are localized mainly on $\mathrm{Al}$ and, and are predominantly $p$ in character. The pseudogap around $-3.8 \mathrm{eV}$ reflects mainly the $s-p$ separation of Al. Overall, the occupied valence bands arise mostly from the $\mathrm{Al} s$ and $p$ states, confirming that $\mathrm{Al}$ is indeed anionic in this compound.

Notice that while the pseudogap (in the vicinity of the Fermi energy) widens with pressure increase, the valence 
band width also increases with pressure [Fig. 8(a)]. For pressures of 0,60 , and $120 \mathrm{GPa}$, the $r_{s}$ values are 2.55, 2.14, and 2.00 , respectively (for $\mathrm{Na}$ and $\mathrm{Al}$ under ordinary conditions, its values would be 3.93 and 2.07, respectively). From a free-electron point of view, we would expect the valence band widths, corresponding to the $r_{s}$ values, to be 7.7, 10.9, and $12.5 \mathrm{eV}$ respectively, which compare very well with the band width from DFT-GGA calculations. Band narrowing under pressure ${ }^{1}$ is not seen in this system up to the maximum pressure considered here. Zone plane activation stabilization arises from the increase in the magnitude of $\langle\mathbf{k}|V(\mathbf{r})| \mathbf{k}+\mathbf{K}\rangle$ alone.

\section{ZONE PLANE ACTIVATION AND ITS RELATION TO BONDING}

Pseudogap widening in the vicinity of the Fermi level associated with the zone plane activation mechanism warrants a more detailed assessment of the bonding patterns near the Fermi level. As we pointed out, the proposed mechanism for stability of $\mathrm{NaAl}$ arises mainly from the lattice potential component $V(220)$ in the nearly free-electron context. In a broader context, the effect of the lattice potential on initially free electrons is the introduction of localized atomiclike states (or eventually Wannier-like states). In the extreme case where we have full nuclear potentials and infinite planewave number, we should begin to reconstruct the full chemistry. By this we mean that we will have initiating atomic orbitals, which, upon inclusion of mutual interactions and adaptation to the translational symmetry, will give rise to crystal orbitals that are linear combination of atomic orbitals.

One essential connection between the two extreme independent approaches to electronic origin of cohesion in the crystalline state (nearly free-electron and localized chemical bonding) is the nodal structure of wave functions, reflecting the symmetry relations between the Hamiltonian and the resultant Bloch states. The $V(220)$ lattice potential component will be associated with a $\mathbf{k}$ vector that satisfies the condition: $\mathbf{k} \cdot \mathbf{K}=K^{2} / 2$, where $\mathbf{K}=(220)$. This leads to the condition that $k_{x}+k_{y}=4 \pi / a$, a condition placing a constraint on the locations of the nodal planes of the planewave $e^{i \mathbf{k} \cdot \mathbf{r}}$ (for both real and imaginary parts), which are the solutions to $\mathbf{k} \cdot\left(\mathbf{r}+\mathbf{r}_{o}\right)$ $=n \pi$ (where $n$ is an integer and $\mathbf{r}_{o}$ is an arbitrary shift).

The importance of the nodal structure lies in the fact that k's are not only the wavevectors of planewaves, but also the labels of the translational symmetry of the Bloch states. In other words, the k's must be such that $e^{i \mathbf{k} \cdot \mathbf{r}}$ transforms as an irreducible representation of the translational symmetry group of the lattice. In particular, if $\mathbf{k}$ and $\mathbf{K}$ are collinear, then for $\mathbf{K}=(220)$, it follows that $\mathbf{k}=(110)$. The nodal planes are then given by the equation $x+y=n a / 2$. Not surprisingly, these are the (220) Miller index planes. In Fig. 9(a), we show a partial unit cell relevant to our bonding analysis (which is further developed below). The two cubes are two of the octants of the full unit cell shown in Fig. 2. The nodal planes corresponding to $\mathbf{k}=(110)$ are shown in Fig. 9(b).

Thus, in a localized chemical picture, the electronic states [linear combinations of atomic orbitals (LCAOs)] corresponding to $\mathbf{k}=(110)$ should reflect the implied nodal

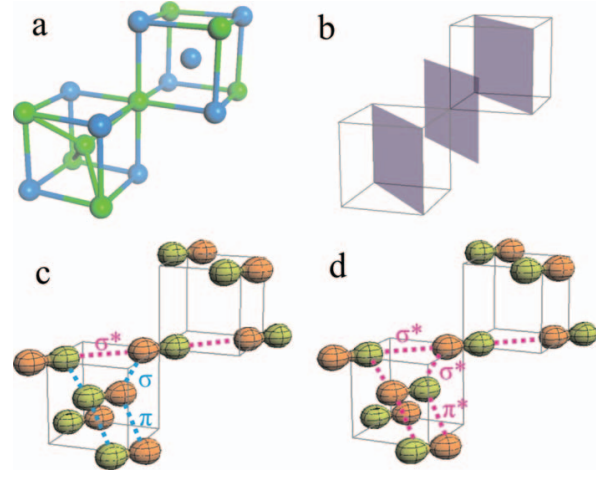

FIG. 9. (a) A partial unit cell of the NaAl structure, showing two of the octants of the full cubic unit cell to emphasize the local bonding environment of Al. (b) Nodal planes corresponding to $\mathbf{k}=(110)$, with the partial structure of part (a) of this figure is shown as two cubic boxes. (c) The orbital at $\mathbf{k}=(110)$, below the Fermi level, which has an overall bonding character. (d) The orbital at $\mathbf{k}=(110)$, above the Fermi level, which has an overall antibonding character. We use a representation for $p$ orbitals of the $\mathrm{L}$ shell $(2 p)$ for clarity, though the true $p$ orbitals should be $3 p$, and have a radial node as well. This simplification should not affect our argument. We use orange and green to represent different phases of the $p$-wave functions. The color-coded dotted lines represent the type of interaction between adjacent $p$ orbitals: blue-bonding and red-antibonding

structure arising from the nearly free-electron approach. ${ }^{58}$ The DFT-GGA calculations clearly show that the states quite near the Fermi level include two sets of threefold degenerate orbitals, corresponding to three sets of distinct (220) Miller planes. In the Wigner-Seitz projection, these band states are entirely associated with $\mathrm{Al}$ and have zero contributions from the valence states of $\mathrm{Na}$. Moreover, these orbitals are completely of $p$ character. Obviously, the absence of $s$ character in the Kohn-Sham wave functions at $\mathbf{k}=(110)$ is a consequence of the presence of the nodal planes, as shown in Fig. 9 (b).

One set of the threefold degenerate orbitals is at energy slightly below the Fermi level and one of these orbitals is shown in Fig. 9(c). We see that the $p$ orbitals of Al in the lower front cube show dominant $p p \pi$-type bonding interaction along the body diagonal (look at the phase relationship of the center orbital with the two orbitals in the bottom face). Judging from the separations for both cubes in Fig. 9(c), the bonding along their faces includes weaker, $p p \sigma$ bonding and $p p \sigma^{*}$ antibonding interactions. The other two orbitals in this threefold degenerate set are similar to this one, with $p$ orbitals oriented perpendicularly to the other two sets of (220) Miller planes. The corresponding crystal orbital at an energy slightly above the Fermi level is shown in Fig. 9(d). In this case, the most important interaction is again of $p p \pi$-type, but antibonding (along the body diagonal of the lower front cube). And as in the former case, the secondary ppo bonding and $p p \sigma^{*}$ antibonding interactions remain present along the faces of the cubes.

As anticipated, at $\mathbf{k}=(110)$, the orbital picture of the electronic states displays the same nodal structure as the free-electron states that participate in the zone plane activation mechanism. In this localized bonding picture, a gap is expected to separate these states due to the dominant $p p \pi$ and $p p \pi^{*}$ interactions. Indeed, in DFT-GGA calculations, there is a small but persistent gap across the Fermi level 
between these Kohn-Sham levels, which increases with pressure. At ordinary pressure, the gap is $41 \mathrm{meV}$. It increases to $49 \mathrm{meV}$ at $20 \mathrm{GPa}$ and then to $64 \mathrm{meV}$ at $60 \mathrm{GPa}$. The growth of the band gap in a LCAO way of thinking is attributable to the decreased interatomic spacing and correspondingly increasing orbital overlaps. This growing gap is consistent with the growth of the pseudogap around the Fermi level, as we have seen in Fig. 8(a). Thus, a bonding analysis based on localized $p$ orbitals reproduces both the symmetry of the Bloch states and also the stabilizing effect of the Jones or higher zone plane type of activation, which was originally proposed within a nearly free-electron theory. A bridge is thus formed between this way of thinking and an orbital picture of chemical bonding.

\section{CONCLUSIONS}

We began with a brief account of the concept of HumeRothery's systematization and empirical rules for stability of metals and intermetallic compounds, as well as the succeeding developments of Jones, and then Mott and Jones, and others. We then extended the Mott-Jones argument to a general zone plane activation approach to accounting for structural selection as the density increases. Here we provide a theoretical basis for the continued utility of the Jones zone concept when pressure becomes a nontrivial thermodynamic variable. Specifically, we have examined how the secondorder band structure energy with a Lindhard form of electronic screening may change with density (suggesting again that the divergence of the slope of the electron screening function near $2 k_{F}$ ) may also have an important effect on any ensuing stability. We have then further extended these notions to pressure-induced Jones zone activation.

As an example, we have demonstrated computationally that an ordered intermetallic compound of $\mathrm{NaAl}$ in the quite striking double-diamond structure can become stabilized by pressure. More importantly, this compound served us as a little laboratory for (a) examining the detailed working of zone plane activation reasoning for structural stability and (b) forging a connection between free-electron, nearly freeelectron, and LCAO pathways.

The case for experimental synthesis of $\mathrm{NaAl}$ with compression therefore seems quite compelling, given our computational results. It may also be of interest from the following points of view. First, the existence or nonexistence of the predicted compound and its actual structure will offer valuable insights regarding our current understanding of structural principles of intermetallic compounds under high pressure and also the accuracy of first-principle calculations in terms of predicting phase stabilities. Second, the existence of $\mathrm{NaAl}$ at moderate compression may prove useful in tailoring the reaction pathways in the decomposition of $\mathrm{Na}-\mathrm{Al}$ hydride systems, as argued earlier. Last, Al is a superconductor at ambient pressure $\left(T_{C}=1.18 \mathrm{~K}\right)$, and its $T_{C}$ decreases with increasing pressure. ${ }^{59}$ Possible superconductivity in $\mathrm{Na}$ at normal and elevated pressures has not so far been established. It will be intriguing to scrutinize the superconductivity, or the absence thereof, of the binary $\mathrm{NaAl}$ system of simple metals under pressure. Might an enhanced electron- ion interaction along with an eventual rise of states near the Fermi level come together in determining the possibility of superconductivity in this compound?

\section{APPENDIX: COMPUTATIONAL METHODS}

We use the DFT approach, ${ }^{60}$ as implemented with planewave basis sets ${ }^{61}$ and the projector-augmented wave method, ${ }^{62}$ within the static lattice approximation. These are applied to study the structural and electronic behavior of proposed $\mathrm{Na}-\mathrm{Al}$ alloys in a pressure range from 0 to 100 $\mathrm{GPa}$, which corresponds to an approximately twofold compression. Exchange-correlation functionals in PerdewBerke-Ernzerhof form ${ }^{63}$ within the GGA (Ref. 64) are utilized throughout. We used two versions of pseudopotentials for sodium. The first version only includes the $3 s$ electrons as the valence set. To account for possible overlapping of core wave functions of $\mathrm{Na}$ as interatomic separation is reduced, ${ }^{4}$ we also use a pseudopotential for sodium treating only the $1 \mathrm{~s}$ electrons as the modeled core states (pseudocore radius $=0.767 \AA$ ), and the $2 s, 2 p, 3 s$, and higher atomic states as the valence set. The first of these forms for the Na pseudopotential cannot reproduce the observed structural preference between bcc and fcc at most pressures, but the second does. However, the difference between the relative enthalpies calculated from the two choices of pseudopotential is small (within $5 \mathrm{meV} /$ atom), and accordingly we use the first form for fast structural preselection, while then comparing the results with those from the second. In view of the high charge on the aluminum core $(+3)$ and hence the relatively small ionic radius, we employ a pseudopotential that treats only the $3 s$ and $3 p$ states as valence, with a small pseudocore radius of $0.899 \AA$.

The total energy evaluation for each structure at a given density is carried out in three steps. First, a given starting structure is preoptimized for a specified external pressure by varying simultaneously the unit cell shape and size and the atomic positions. The external pressure is set by adding a constant stress to the diagonal elements of the stress tensor and then minimizing the enthalpy. Second, the preoptimized structure is subsequently reoptimized at constant density, minimizing the total energy with respect to cell parameters (at constant volume) and ion positions.

We use the Monkhorst-Pack scheme ${ }^{65}$ of k-point sampling for the zone sums, and select the number of $\mathbf{k}$ points to achieve a convergence in total energy within $\sim 1 \mathrm{meV} /$ atom. In the final step, the total energy of each optimized structure is calculated again with the more accurate tetrahedron zone summation with Blöchl correction. ${ }^{66}$ All calculations using the $\mathrm{Na}$ pseudopotential that include a supposed set of core states in the valence set are carried out with a planewave cutoff of $1200 \mathrm{eV}$. Calculations with the valence-only pseudopotential use a cutoff of $500 \mathrm{eV}$.

\section{ACKNOWLEDGMENTS}

Our work at Cornell was supported by NSF Research Grant Nos. CHE-0613306 and DMR-0907425. This research used resources of the National Energy Research Scientific Computing Center, which is supported by the Office of Sci- 
ence of the U.S. Department of Energy under Contract No. DE-AC02-05CH11231. Particular acknowledgment is also made for the use of the Research Computing Facilities at the Cornell Center for Materials Research.

${ }^{1}$ J. B. Neaton and N. W. Ashcroft, Nature (London) 400, 141 (1999).

${ }^{2}$ M. Hanfland, K. Syassen, N. E. Christensen, and D. L. Novikov, Nature (London) 408, 174 (2000).

${ }^{3}$ I. Tamblyn, J.-Y. Raty, and S. A. Bonev, Phys. Rev. Lett. 101, 075703 (2008).

${ }^{4}$ J. B. Neaton and N. W. Ashcroft, Phys. Rev. Lett. 86, 2830 (2001).

${ }^{5}$ J.-Y. Raty, E. Schwegler, and S. A. Bonev, Nature (London) 449, 448 (2007)

${ }^{6}$ E. Gregoryanz, L. F. Lundegaard, M. I. McMahon, C. Guillaume, R. J. Nelmes, and M. Mezouar, Science 320, 1054 (2008).

${ }^{7}$ J. Feng, R. G. Hennig, N. W. Ashcroft, and R. Hoffmann, Nature (London) 451, 445 (2008)

${ }^{8}$ Y. Ma, M. Eremets, A. R. Oganov, Y. Xie, I. Trojan, S. Medvedev, A. O. Lyakhov, M. Valle, and V. Prakapenka, Nature 458, 182 (2009).

${ }^{9}$ T. Matsuoka, M. Debessai, J. J. Hamlin, A. K. Gangopadhyay, and J. S. Schilling, Phys. Rev. Lett. 100, 197003 (2008).

${ }^{10}$ B. Rousseau and N. W. Ashcroft, Phys. Rev. Lett. 101, 046407 (2008).

${ }^{11}$ C. C. Fischer, K. J. Tibbetts, D. Morgan, and G. Ceder, Nature Mater. 5, 641 (2006).

${ }^{12}$ C. W. Glass, A. R. Oganov, and N. Hansen, Comput. Phys. Commun. 175, 713 (2006)

${ }^{13}$ C. J. Pickard and R. J. Needs, Phys. Rev. Lett. 97, 045504 (2006).

${ }^{14}$ G. J. Ackland and I. R. Macleod, New J. Phys. 6, 138 (2004)

${ }^{15}$ V. F. Degtyareva, Phys. Usp. 49, 369 (2006).

${ }^{16}$ V. F. Degtyareva and O. Degtyareva, New J. Phys. 11, 063037 (2009).

${ }^{17}$ H. Jones, Proc. R. Soc. London, Ser. A 144, 225 (1934).

${ }^{18}$ N. F. Mott and H. Jones, The Theory of the Properties of Metals and Alloys (Oxford University Press, Oxford, 1936).

${ }^{19}$ R. Hoffmann and R. B. Woodward, Acc. Chem. Res. 1, 17 (1968).

${ }^{20}$ H. Jahn and E. Teller, Proc. R. Soc. London, Ser. A 161, 220 (1937).

${ }^{21}$ W. Hume-Rothery, J. Inst. Met. 35, 295 (1926).

${ }^{22} \mathrm{H}$. Jones, The Theory of Brillouin Zone and Electronic States in Crystals, 2nd ed. (North Holland, Amsterdam, 1975).

${ }^{23}$ A. Blandin, in Phase Stability in Metals and Alloys, edited by P. S. Rudman, J. Stringer, and R. I. Jaffee (McGraw-Hill, New York, 1966).

${ }^{24}$ V. Heine, in Phase Stability in Metals and Alloys, edited by P. S. Rudman, J. Stringer, and R. I. Jaffee (McGraw-Hill, New York, 1966).

${ }^{25}$ D. Stroud and N. W. Ashcroft, J. Phys. F: Met. Phys. 1, 113 (1971).

${ }^{26}$ D. Stroud and N. W. Ashcroft, in Solid State Physics, edited by H. Ehrenreich, F. Seitz, and D. Turnbull (Academic, New York, 1978), Vol. 33.

${ }^{27}$ N. W. Ashcroft, Phys. Rev. 140, A935 (1965).

${ }^{28}$ N. W. Ashcroft, Phys. Rev. B 39, 10552 (1989).

${ }^{29}$ N. W. Ashcroft, Phys. Rev. B 19, 4906 (1979).

${ }^{30}$ A. T. Paxton, M. Methfessel, and D. G. Pettifor, Proc. R. Soc. London, Ser. A 453, 1493 (1997).

${ }^{31}$ The concepts of translationengleiche and klassengleiche subgroups of space groups are discussed in H. Wondratschek, "General introduction to the subgroups of spaces groups," in International Tables for Crystallography, edited by H. Wondratschek and U. Müller (Kluwer, Dordrecht, 2008), Vol. A1, pp. 6-23.

${ }^{32}$ M. H. Cohen, in Metallic Solid Solutions, edited by J. Friedel and A. Guinier, (Benjamin, New York, 1963).

${ }^{33}$ The ion-sphere model supposes that the charge of valence electrons is uniformly distributed through a sphere of radius $r_{s}$ about each ion, while neglecting any overlap between these electron spheres.

${ }^{34}$ N. W. Ashcroft and D. C. Langreth, Phys. Rev. 155, 682 (1967).

${ }^{35} \mathrm{~W}$. A. Harrison, Pseudopotentials in the Theory of Metals (Benjamin, New York, 1966).

${ }^{36}$ E. Zintl, Angew. Chem. 52, 1 (1939); Chemistry, Structure, and Bonding of Zintl Phases and Ions, edited by S. M. Kauzlarich (VCH, New York, 1996).

${ }^{37}$ H. Schäfer, B. Eisenmann, and W. Müller, Angew. Chem., Int. Ed. Engl. 12, 694 (1973).

${ }^{38}$ Z. Dong and J. D. Corbett, J. Am. Chem. Soc. 115, 11299 (1993).

${ }^{39}$ J. Evers and G. Oehlinger, Inorg. Chem. 39, 628 (2000).

${ }^{40}$ The two metals only show slight solubility in each other in the liquid state at above $659^{\circ} \mathrm{C}$, see T. B. Massalski, Binary Alloy Phase Diagrams (ASM International, Materials Park, OH, 2000).

${ }^{41}$ E. Yu Tonkov, Compounds and Alloys under High Pressure: A Handbook (CRC, Boca Raton, 1998).

${ }^{42}$ J. K. Kang, J. Y. Lee, R. P. Muller, and W. A. Goddard III, J. Chem. Phys. 121, 10623 (2004).

${ }^{43}$ J. E. Inglesfield, J. Phys. C 4, 1003 (1971).

${ }^{44}$ M. B. McNeil, W. B. Pearson, L. H. Bennett, and R. E. Watson, J. Phys. C 6, 1 (1973).

${ }^{45}$ This implies that the free-electron sphere intersects the specified zone planes, and yet the large zone is not entirely inside the sphere; that is, the Fermi sphere is somewhere between "inscribed" and "inscribing" surfaces relative to the large zone.

${ }^{46}$ W. Kraus and G. Nolze, J. Appl. Crystallogr. 29, 301 (1996).

${ }^{47}$ N. W. Ashcroft, J. Phys. C 1, 232 (1968).

${ }^{48}$ J. Feng, "Theoretical aspects of light-element alloys under extremely high pressure," Ph.D. thesis, Cornell University, 2007; J. Feng, N. W. Ashcroft, and R. Hoffmann, Phys. Rev. Lett. 98, 247002 (2007).

${ }^{49} \mathrm{We}$ also optimized the structure in a fourfold supercell with 16 atoms at $\sim 20,40,60$ and $80 \mathrm{GPa}$, without any point-group in the starting structure or any symmetry constraint. The resultant structures show very small distortions from the cubic double-diamond structure. With a $0.1 \AA$ A tolerance, all of these supercells are identical to the cubic double-diamond structure. The energy difference due these small distortions is negligibly small.

${ }^{50}$ See supplementary material at http://dx.doi.org//10.1063/1.3328198 for the auxiliary materials.

${ }^{51}$ D. G. Pettifor, in Physical Metallurgy, edited by R. W. Cahn and P. Haasen (North-Holland, Amsterdam, 1983), Chap. 3; D. G. Pettifor, Solid State Commun. 51, 31 (1984).

${ }^{52}$ P. C. Schmidt, Phys. Rev. B 31, 5015 (1985).

${ }^{53}$ N. E. Christensen, Phys. Rev. B 32, 207 (1985).

${ }^{54}$ J. Köhler, S. Deng, C. Lee, and M.-H. Whangbo, Inorg. Chem. 46, 1957 (2007).

${ }^{55}$ A. Zunger, Phys. Rev. B 17, 2582 (1978).

${ }^{56}$ R. Hoffmann, J. Chem. Phys. 39, 1397 (1963).

${ }^{57}$ T. Hughbanks and R. Hoffmann, J. Am. Chem. Soc. 105, 3528 (1983).

${ }^{58}$ For a detailed discussion of the relationship of LCAO and nearly freeelectron states, see R. Berger, S. Lee, and R. Hoffmann, "Using linear combination of atomic orbitals to understand Hume-Rothery' electroncounting rule in complex phases: From 1-D to gamma-brass," (to be published).

${ }^{59}$ D. U. Gubser and A. W. Webb, Phys. Rev. Lett. 35, 104 (1975).

${ }^{60}$ P. Hohenberg and W. Kohn, Phys. Rev. 136, 864B (1964); W. Kohn and L. J. Sham, ibid. 140, 1133A (1965)

${ }^{61}$ All calculations are performed with the Vienna $a b$ initio simulations package (VASP), see G. Kresse and J. Furthmller, Comput. Mater. Sci. 6, 15 (1996); Phys. Rev. B 54, 169 (1996).

${ }^{62}$ P. P. Blöchl, Phys. Rev. B 50, 17953 (1994); G. Kresse and D. Joubert, ibid. 59, 1758 (1999).

${ }^{63}$ J. P. Perdew, K. Burke, and M. Ernzerhof, Phys. Rev. Lett. 77, 3865 (1996).

${ }^{64}$ D. C. Langreth and M. J. Mehl, Phys. Rev. B 28, 1809 (1983); A. D. Becke, Phys. Rev. A 38, 3098 (1988).

${ }^{65}$ H. J. Monkhorst and J. D. Pack, Phys. Rev. B 13, 5188 (1976).

${ }^{66}$ P. E. Blöchl, O. Jepsen, and O. K. Andersen, Phys. Rev. B 49, 16223 (1994). 